\title{
Micro-fabricated channel with ultra-thin yet ultra-strong windows enables electron microscopy under 4-bar pressure
}

\author{
Tuncay Alan, ${ }^{1,2, a)}$ Tadahiro Yokosawa, ${ }^{3}$ João Gaspar, ${ }^{4,5}$ Gregory Pandraud, ${ }^{1}$ Oliver Paul, ${ }^{4}$ \\ Fredrik Creemer, ${ }^{1}$ Pasqualina M. Sarro, ${ }^{1}$ and Henny W. Zandbergen ${ }^{3}$ \\ ${ }^{1}$ Delft Institute of Microsystems and Nanoelectronics, Delft University of Technology, 2628 CT Delft, \\ The Netherlands \\ ${ }^{2}$ Department of Mechanical and Aerospace Engineering, Monash University, Clayton, 3800 VIC, Australia \\ ${ }^{3}$ Centre for High Resolution Electron Microscopy, Delft University of Technology, 2628 CJ Delft, \\ The Netherlands \\ ${ }^{4}$ Department of Microsystems Engineering (IMTEK), University of Freiburg Georges-Kohler-Allee 103, \\ D-79110 Freiburg, Germany \\ ${ }^{5}$ International Iberian Nanotechnology Laboratory (INL), Avenida Mestre Jose Veiga, 4715-330 Braga, \\ Portugal
}

(Received 14 November 2011; accepted 6 February 2012; published online 23 February 2012)

\begin{abstract}
Transmission electron microscopy (TEM) of (de-)hydrogenation reactions is crucial to characterize efficiency of hydrogen storage materials. The nanoreactor, a micromachined channel with 15-nm-thick windows, effectively confines the gas flow to an electron-transparent chamber during TEM of reactions. Realistic experiments require very high pressures to be sustained by the device. Nanomechanical bulge tests and simulations show that due to a very strong size effect, ultra-thin device components can reliably withstand tensile stresses as high as $19.5 \mathrm{GPa}$ enabling high pressure operation. We use the device to characterize Pd particles under a 4-bar $\mathrm{H}_{2}$ pressure within the ultra-high-vacuum of the TEM. (C) 2012 American Institute of Physics. [http://dx.doi.org/10.1063/1.3688490]
\end{abstract}

The increasing demand in alternative, clean energy sources has attracted a considerable attention to hydrogen as energy carrier and nanostructured metal hydrides as potential hydrogen storage materials. ${ }^{1-3}$ To evaluate the performance of candidate materials, structural changes taking place during hydrogenation and dehydrogenation process should be characterized at atomic resolution and under realistic conditions. Environmental TEM (E-TEM) enables real-time, in-situ imaging of chemical reactions below the 20 millibar threshold. ${ }^{4}$ However, the onset of reactions may require much higher pressures, ${ }^{5}$ hence greatly limiting the applicability of E-TEM. In this letter, we show that TEM of reactions under very high pressures can indeed be accommodated with the help of the nanoreactor, an external, microfabricated device which confines the gas flow and reactions to an electron transparent microchannel within the TEM chamber. The nanoreactor (Fig. 1(a)) consists of two micro-machined parts brought into contact to form a channel. Continuous injection and extraction of $\mathrm{H}_{2}$ gas are made possible via two openings at the bottom chip. The geometry of the device presented here differs slightly from an earlier version: ${ }^{6,7}$ at the centre of each chip, there is a $1.2 \mu$ m-thick SiN membrane containing an array of 15-nm-thick, circular, transparent windows, connected to the thicker membrane through smoothed corners, free of stress singularities.

The ultra-thin windows on both sides are aligned on top of each other so as to effectively transmit the electron beam through the channel and enable imaging while reactions take place (Fig. 1(b)). Clearly, higher quality images are possible for window thicknesses approaching zero. Yet, there is a

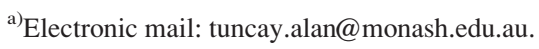

compromise between image quality and operational limits of the device. ${ }^{8}$ For the reactor to operate safely, the chamber ceilings $(1.2-\mu \mathrm{m}$-thick) and each of the ultrathin windows (15-nm-thick) must survive the pressure difference between the vacuum of the microscope and the pressurized gas inside the channel (Fig. 1(c)). Mechanical failure of a single window, broken during operation would result in high pressure gas to be released into the high-vacuum chamber and could significantly damage the electron source. Mechanical properties of materials depend critically on process parameters and dimensional scales (higher strengths are expected for smaller sizes). ${ }^{9-11}$ The nanoreactor is a multi-scale device with electron transparent windows that are 100 times thinner than the chamber ceilings. What are the maximum pressures which

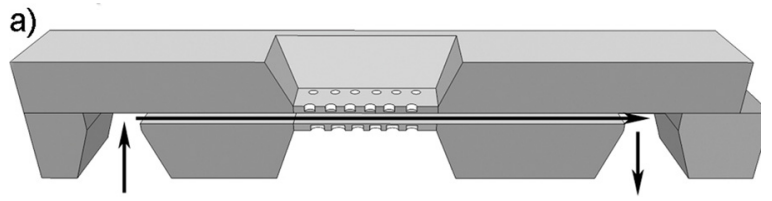

b)
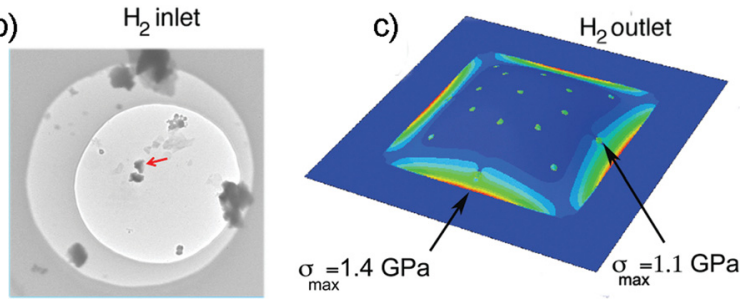

FIG. 1. (Color online) (a) Schematic of the MEMS nanoreactor, (b) closeup view of a particle enclosed between two circular electron transparent windows implemented in the new generation device, (c) stress distribution within the $1.2 \mu \mathrm{m}$-thick central square plate containing circular windows under 4 bar pressure difference, calculated by finite element simulations. 
can be sustained by the thick chamber ceilings and ultra-thin windows during operation? And, could we exploit any size effect to improve the device performance? To address these questions and to establish the mechanical reliability of the device, elastic properties and fracture strength statistics of similarly prepared $\mathrm{SiN}$ membranes with thicknesses ranging from $15 \mathrm{~nm}$ to $1 \mu \mathrm{m}$ were characterized through nanomechanical plane-strain bulge tests. ${ }^{12}$ Deflections and stress distributions within different device components under increasing pressures were simulated using the elastic constants determined from the experiments. Fracture probabilities corresponding to maximum stresses were then inferred by comparing the simulated stresses to the experimental failure statistics.

Test samples consisted of rectangular free standing thin films with varying thicknesses and lateral dimensions $\left(0.2 \times 2 \mathrm{~mm}^{2}, 0.9 \times 9 \mathrm{~mm}^{2}\right)$. SiN films with thicknesses of $15,70,245$, and $1023 \mathrm{~nm}$ were deposited on oxidized (100)oriented $\mathrm{Si}$ wafers through low pressure chemical vapor deposition (LPCVD). The deposited films were covered by a protective $\mathrm{SiO}$ layer. Wafers were patterned on their backsides and etched through by a $33 \% \mathrm{KOH}$ solution, thus defining the membranes. As a final step, the samples were released by immersing the wafers in a 7:1 buffered hydrofluoric acid (HF) solution. The fabrication steps are summarized in the inset of Fig. 2. Wafers with released membranes were glued on separate chucks containing individual pressure feed-throughs for each membrane and the chucks were mounted on the custom-built bulge test set-up. ${ }^{12}$ After the dimensions of each membrane were measured with an optical scan (exploiting the difference of the optical reflectivity of the membranes and surrounding area), a uniform pressure was incrementally applied in steps of $5 \mathrm{kPa}$ from the membrane backside. Throughout the experiment, the deflected membrane profile was measured at each pressure level and the maximum out-of-plane deflection at the center of the membrane was recorded with a resolution of $10 \mathrm{~nm}^{12}$

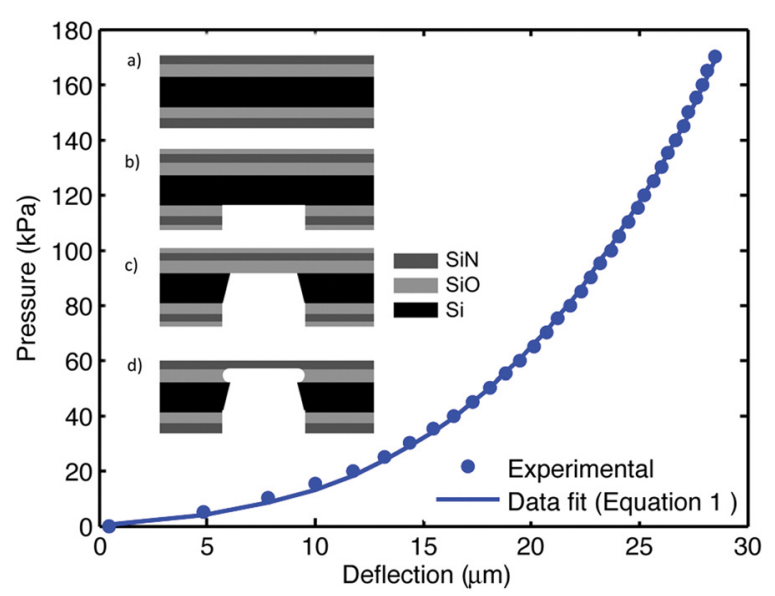

FIG. 2. (Color online) Deflection of a $1.023 \mu \mathrm{m}$-thick rectangular SiN film under increasing uniform pressure. Experimental pressure-deflection data were fit by Eq. (1) to estimate plane-strain elastic modulus presented in Table I (the membrane has failed at 1.7 bars). Inset: Fabrication of test samples: (a) a thin layer of $\mathrm{SiN}$ was deposited on an oxidised $\mathrm{Si}$ wafer, (b) protective $\mathrm{SiO}$ layer was deposited and the wafer backside was patterned, (c) the wafers were etched in a $33 \% \mathrm{KOH}$ solution and the membranes were released in a buffered HF solution.
Repeating this procedure until fracture, the pressuredeflection curve and the maximum fracture load were determined for all of the tested structures.

During loading, the samples undergo deflections that are up to three orders of magnitude larger than the film thickness and, as such, the pressure-deflection curves are dominated by the membrane effects. Similarly, the high length-to-width ratios of the tested membranes ensure that the membranes respond to the out-of-plane differential pressures by a plane strain deformation. ${ }^{12,13}$ As illustrated in the inset of Fig. 3, the central part of the membrane deforms into a cylinder-like shape that does not depend on the position along the long axis. Hence, the experimental data were analyzed using a plane strain model ${ }^{12,14}$ that considers the dominant membrane stresses, as well as the bending stresses at the supporting edges. ${ }^{14}$ Accordingly, the out-of-plane deflection, $w$, and the effective line force, $S$, which builds up within the membrane with increasing deflections, are given by equations (1) and (2) respectively.

$$
\begin{aligned}
w(x)= & \frac{P a^{2}}{2 S}\left(\frac{1}{4}-\frac{x^{2}}{a^{2}}\right) \\
& -\frac{\frac{P}{S}\left(D_{2}+\frac{K a}{2}\right)\left[\cosh \frac{a}{2} \sqrt{\frac{S}{D_{2}}}-\cosh \left(\sqrt{\frac{S}{D_{2}}} x\right)\right]}{S \cosh \left(\frac{a}{2} \sqrt{\frac{S}{D_{2}}}\right)+K \sqrt{\frac{S}{D_{2}}} \sinh \left(\frac{a}{2} \sqrt{\frac{S}{D_{2}}}\right)}, \\
S= & S_{0}+\frac{D_{2}}{a^{2}} \sum_{i=1}^{3}\left(\frac{P a^{4}}{\sqrt{\frac{\left(D_{2}\right)^{3}}{D_{0}}}}\right)^{i-1} \\
\times & \frac{\left[\beta_{i, 1}+\beta_{i, 2} \cosh \left(\sqrt{\frac{S}{D_{2}}} a\right)+\beta_{i, 3} \sinh \left(\sqrt{\frac{S}{D_{2}}} a\right)\right]}{\left[\frac{S a^{2}}{D_{2}} \cosh \left(\frac{a}{2} \sqrt{\frac{S}{D_{2}}}\right)+\left(\frac{K a^{2}}{D_{2}}\right) \sqrt{\frac{S}{D_{2}}} \sinh \left(\frac{a}{2} \sqrt{\frac{S}{D_{2}}}\right)\right]^{2}},
\end{aligned}
$$

respectively. Here, $P$ is the applied pressure, $a$ is the width of the plate, and $S_{0}=\sigma_{0} h$, where $\sigma_{0}$ is the residual stress in the thin film. Elastic parameters are the bending stiffness $D_{2}=\frac{E h^{3}}{12\left(1-\nu^{2}\right)}$, stretching stiffness $D_{0}=\frac{E h}{1-\nu^{2}}$, and the torsional stiffness $K=\frac{E h^{2}}{0.78\left(1-\nu^{2}\right)}$, where $E, \nu$, and $h$ denote the elastic modulus, Poisson's ratio, and the film thickness,

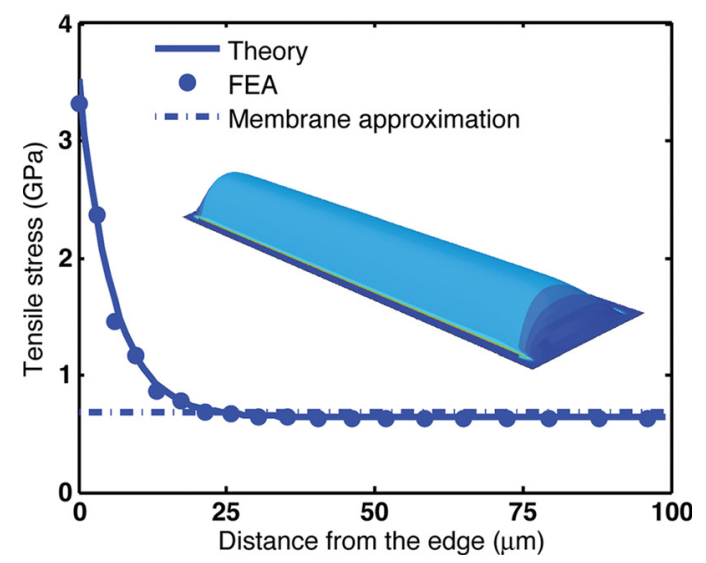

FIG. 3. (Color online) Stress distribution near the edges of a $1.023-\mu \mathrm{m}$-thick rectangular $\mathrm{SiN}$ film, which fractured under 1.7 bar pressure (marked with an arrow in Fig. 4) calculated by standard membrane approximation, FEA, and Eq. (3) (theory). Inset: Deflected shape of the film right before fracture. 


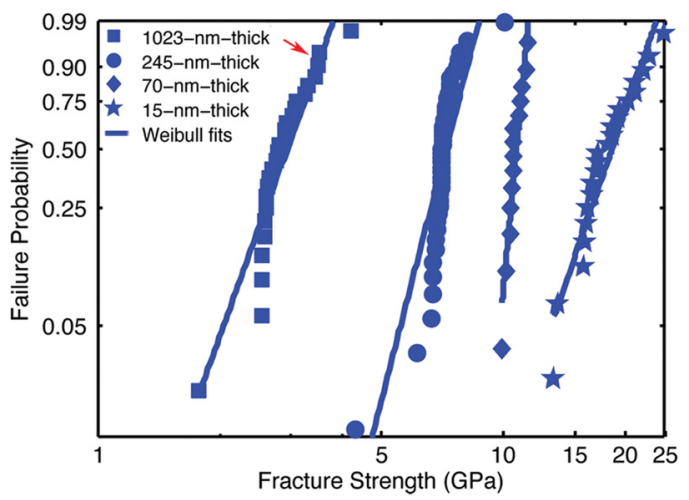

FIG. 4. (Color online) Weibull strength distribution for rectangular membranes with thicknesses decreasing from $1023 \mathrm{~nm}$ to $15 \mathrm{~nm}$. Corresponding Weibull strengths and elastic moduli are summarized in Table I.

respectively. The coefficients $\beta_{i, j}(j=1,2,3)$ as function of the above variables and further details of the model are documented elsewhere. ${ }^{12}$

After the tests, plane strain elastic moduli and residual stresses of the deposited films were determined by fitting the set of equations (1) and (2) to the experimental pressuredeflection curve for rectangular membranes. Finally, for each tested sample, the stress distribution corresponding to the fracture load was calculated as

$$
\sigma(x, z)=\sigma_{0}+\frac{E}{2 a\left(1-\nu^{2}\right)} \int_{-a / 2}^{a / 2}\left(\frac{d w}{d x}\right)^{2} d x-\frac{E h / 2}{1-\nu^{2}} \frac{d^{2} w}{d x^{2}},
$$

and the maximum tensile stress value within each membrane was inferred as the fracture strength of the sample. Fig. 3 compares the stress distribution in a bulged thin film sample, as calculated by the membrane approximation, finite element analysis (FEA), and Eq. (3). FEA results are in excellent agreement with Eq. (3), whereas the membrane approximation does not take into account the transition near the membrane supports and, hence, underestimates the stresses near the edges.

Weibull strength statistics extracted from all the bulge tests are plotted in Fig. 4. We do not observe a considerable size dependence in elastic properties, apart from a slight drop at the lowest thickness. Similarly, the residual stress remains tensile except for the thinnest film where a compressive stress is observed. However, there is a very strong dependence between sample size and mechanical performance (generally attributed to the lower number of critical defects at smaller volumes ${ }^{15}$ ). The experimental results show that Weibull strength increases 6-fold from 3.1 to $19.5 \mathrm{GPa}$ as the
TABLE I. Extracted material properties.

\begin{tabular}{lccc}
\hline \hline $\begin{array}{l}\text { Thickness } \\
\mathrm{h}(\mathrm{nm})\end{array}$ & $\begin{array}{c}\text { Plane strain } \\
\text { Modulus (GPa) }\end{array}$ & $\begin{array}{c}\text { Residual } \\
\text { Stress (MPa) }\end{array}$ & $\begin{array}{c}\text { Weibull } \\
\text { Strength (GPa) }\end{array}$ \\
\hline 15 & 240 & -135 & 19.5 \\
70 & 277 & 120 & 11 \\
245 & 276 & 139 & 7.4 \\
1023 & 272 & 116 & 3.1 \\
\hline \hline
\end{tabular}

sample thickness decreases from one micrometer down to few nanometers. In order to assess the mechanical limits of the device, the stress distribution within a $1.2-\mu \mathrm{m}$-thick, $200 \mu \mathrm{m}$-wide square plate containing 5 - $\mu$ m-diameter, $15-\mathrm{nm}$ thick circular windows was characterized through a nonlinear finite element simulation (as illustrated in Fig. 1(c)) considering the fundamental mechanical properties summarized in Table I. The analysis was performed with the commercial software ABAQus using 8-node quadratic thin shell elements, S8R5. Under a 4 bar pressure difference, the maximum tensile stress at the supporting edges of the plate is $1.4 \mathrm{GPa}$; similarly, the ultra-thin windows are subject to a maximum stress of $1.1 \mathrm{GPa}$. In the present nanoreactor design sharp corners at the window supports were minimized through a wet etching step (inset Fig. 2(c)) to avoid stress singularities which could compromise the mechanical reliability. Hence, local stress concentrations at the corners were ignored in the analysis. In view of the Weibull strength statistics in Fig. 4, the device is expected to survive the applied pressures $99 \%$ of the time. Our results suggest that a combination of size effects and structural scaling ensures that the ultra-thin (approximately 50-atom-thick) device components can sustain higher pressures with a significantly higher survival rate than their bulkier (1.2- $\mu$ m-thick) counterparts. For higher pressures approaching $10 \mathrm{bar}$, the ultra-thin windows continue to have a survival probability of $99 \%$, while the thicker nanoreactor ceilings become more critical with an estimated survival probability of $75 \%$. This interesting result implies that at higher pressures device failure is mostly related to the thick components while the ultra-thin windows, the most crucial components for higher quality images, remain intact.

To demonstrate the applicability of the nanoreactor for high pressure operation, the assembled new generation device was used to characterize Pd particles under a 4 bar pressure difference and at elevated temperatures. Fig. 5 shows a TEM image of the nanoparticles and the selected area electron diffraction patterns (SAED) of the nano particles under 4 bar $\mathrm{H}_{2}$ pressures at 25 and $240^{\circ} \mathrm{C}$, respectively. When the system temperature is increased from 25 to $240^{\circ} \mathrm{C}$, the

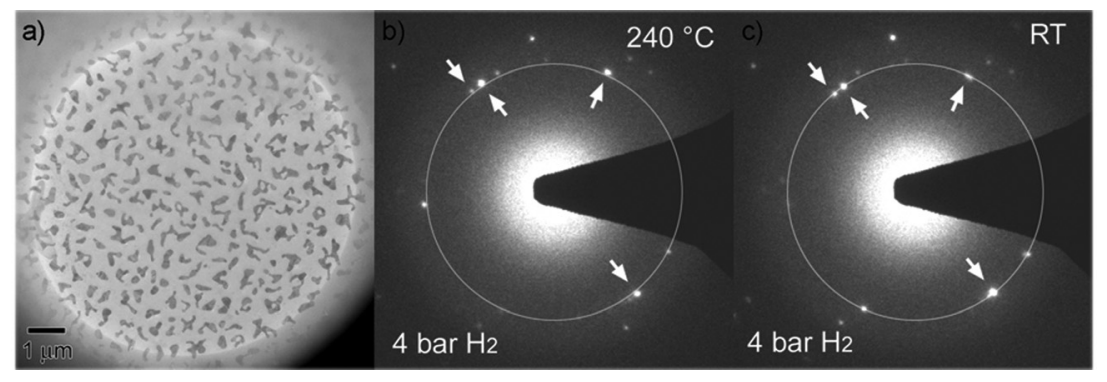

FIG. 5. (a) TEM image of Pd particles under 4 bar $\mathrm{H}_{2}$ pressure. Electron diffraction patterns at 4 bars and (b) $25^{\circ} \mathrm{C}$ and (c) $240^{\circ} \mathrm{C}$. Increasing the temperature results in dehydrogenation, hence reducing the lattice coefficient. A video of the changes in the SAED patterns is presented (enhanced online) [URL: http://dx.doi.org/ 10.1063/1.3688490.1]. 
lattice shrinks due to dehydrogenation and the reflections located on the (022) diffraction ring (indicated by the white circle in the SAED pattern) shift to higher angles. ${ }^{16}$ Similarly, hydrogenation causing the lattice expansion was also confirmed for the reverse case when the temperature is reduced from 240 to $25^{\circ} \mathrm{C}$.

The nanoreactor was shown to reliably operate under 4-bar-pressure. However, it should be noted that the operational limits of the device may further be improved to much higher pressures by exploiting the discussed scaling effect and implementing optimized device geometries.

This research was performed as a part of the Sustainable Hydrogen Program of Advanced Chemical Technologies for Sustainability (ACTS), funded by Netherlands Organisation for Scientific Research (NWO).

${ }^{1}$ B. Sakintuna, F. Lamari-Darkrim, and M. Hirscher, Int. J. Hydrogen Energy 32(9), 1121 (2007).

${ }^{2}$ L. Schlapbach and A. Zuttel, Nature 414, 353 (2001).
${ }^{3}$ S. Isobe, A. Ono, H. Yao, Y. Wang, N. Hashimoto, and S. Ohnuki, Appl. Phys. Lett. 96, 223109 (2010).

${ }^{4}$ R. Sharma and Z. Iqbal, Appl. Phys. Lett. 84, 990 (2004).

${ }^{5}$ P. H. L. Notten, J. L. C. Daams, A. E. M. De Veirman, and A. A. Staals, J. Alloys Compd. 209, 85 (1994).

${ }^{6}$ J. F. Creemer, S. Helveg, G. H. Hoveling, S. Ullmaan, A. M. Molenbroek, P. M. Sarro, and H. W. Zandbergen, Ultramicroscopy 108(9), 993 (2008).

${ }^{7}$ J. F Creemer, S. Helveg, P. J. Kooyman, A. M. Molenbroek, H. W. Zandbergen, P. M. Sarro, J. Microelectromech. Syst. 19(2), 254 (2010).

${ }^{8}$ R. Ramachandra, H. Demers, and N. de Jonge, Appl. Phys. Lett. 98, 093109 (2011).

${ }^{9}$ S. Kumar, M. A. Haque, and H. Gao, Appl. Phys. Lett. 94, 253104 (2009).

${ }^{10}$ T. Alan and A. T. Zehnder, Int. J. Fract. 148(2), 129 (2007).

${ }^{11}$ T. Alan, A. T. Zehnder, D. Sengupta, and M. A. Hines, Appl. Phys. Lett. 89, 231905 (2006).

${ }^{12} \mathrm{O}$. Paul and J. Gaspar, in Reliability of MEMS, edited by O. Tabata and T. Tsuchiya (Wiley-VCH, Weinheim, 2007), pp. 67-122.

${ }^{13}$ Y. Xiang, X. Chen, and J. J. Vlassak, J. Mater. Res. 20(9), 2360 (2005).

${ }^{14}$ V. Ziebart, O. Paul, U. Munch, J. Schwizer, and H. Baltes, J. Microelectromech. Syst. 7, 320 (1998).

${ }^{15}$ A. McCarty and I. Chasiotis, Thin Solid Films 515, 3267 (2007).

${ }^{16}$ T. Yokosawa, T. Alan, G. Pandraud, B. Dam, and H. Zandbergen, Ultramicroscopy 112(1), 47 (2012). 